\title{
SOME EFFECTS OF PARALLEL ENERGY PROPAGATION ON THE STRUCTURE OF DISSIPATIVE TRAPPED ELECTRON MODES
}

\section{PLASMA PHYSICS LABORATORY}

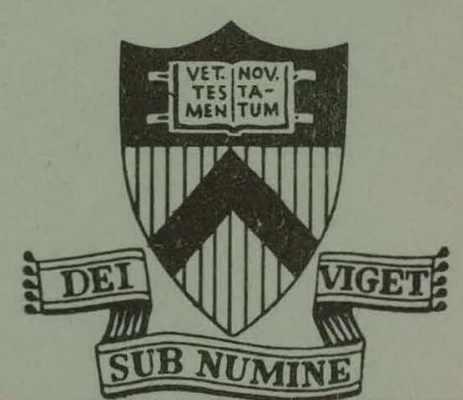

\section{PRINCETON \\ UNIVERSITY PRINCETON, NEW JERSEY}

This work was supported by U. S. Energy Research and Development Administration Contract $\mathrm{E}(11-1)-3073$. Reproduction, translation, publication, use and disposal, in whole or in part, by or for the United States Government is permitted. 


\section{DISCLAIMER}

This report was prepared as an account of work sponsored by an agency of the United States Government. Neither the United States Government nor any agency Thereof, nor any of their employees, makes any warranty, express or implied, or assumes any legal liability or responsibility for the accuracy, completeness, or usefulness of any information, apparatus, product, or process disclosed, or represents that its use would not infringe privately owned rights. Reference herein to any specific commercial product, process, or service by trade name, trademark, manufacturer, or otherwise does not necessarily constitute or imply its endorsement, recommendation, or favoring by the United States Government or any agency thereof. The views and opinions of authors expressed herein do not necessarily state or reflect those of the United States Government or any agency thereof. 


\section{DISCLAIMER}

Portions of this document may be illegible in electronic image products. Images are produced from the best available original document. 


\title{
Some Effects of Parallel Energy Propagation on the structure of \\ Dissipative Trapped Electron Modes
}

\author{
N. R. Sauthoff \\ Plasma Physics Laboratory, Princeton University \\ Princeton, New Jersey 08540
}

\begin{abstract}
The limitation of ballooning by parallel energy propagation is investigated for the dissipative trapped electron mode, wherein the local energy influx is proportional to the poloidally dependent trapped particle fraction. For small energetic asymmetries $\left[\mathrm{mq}<2.5\left(\Omega_{i} \mathrm{a} / \mathrm{c}_{\mathrm{s}}\right)(\mathrm{a} / \mathrm{R})^{3 / 4}\right.$ ] the structure. along the field line is predominantly a phase shift, dictated by the condition that the asymmetric energy input be compensated by parallel energy flow. For large asymmetry, mode number, and parallel arc length, the phase and amplitude variations necessitate an integral equation treatment.
\end{abstract}

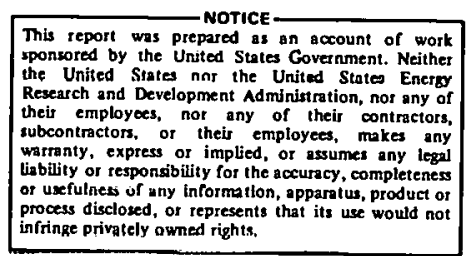


The successful confinement of plasmas of thermonuclear interest is contingent on the restriction of the transport due to collective processes. Local analyses of the fluctuations indicate that the dissipation (both resonant and collisional) in electrons trapped in magnetic field minima destabilizes drift waves, significantly increasing the transport. ${ }^{I}$ In this article we consider the structure and the elementary stability of the modes both because the growth is dependent on the localization and because the experimental study of saturated waves demands an observable means for the analysis of energetics.

The poloidal structure of dissipative trapped electron modes is determined by a competition between a localized energy input from the trapped electrons and parallel energy propagation due to divergence of the parallel ion flow (i.e., the ion sound term). Recent calculations ${ }^{2}$ which treat the integral equation (due to the electron bounce-average of the potential) and find mode structures poloidally localized near the minimum of the magnetic field amplitude neglect several effects: (1) the parallel propagation of energy, which tends to make the amplitude constant along the field line, and (2) poloidal periodicity in a sheared magnetic field, which invalidates the flute approximation and introduces a poloidally dependent damping due to the divergence of the radial energy $f_{l u x}{ }^{3}$ in cases where the mode cannot be poloidally localized $\left(1 q^{\prime}<k_{r}\right)$. In this work we calculate the phase and amplitude variations along the magnetic field 
due to an asymmetry of the energetics. The ion sound energy propagation term is included but the integral nature of the equation is ignored under the assumption that the parallel phase shift is small; the determination of the value of the energetic asymmetry under which this assumption is satisfied is the goal of this paper.

The calculation of the structure of trapped electron modes in toroidally symmetric systems involves the solution of a two-dimensional integro-differential equation of the form 4

$$
\begin{aligned}
& \left(\frac{c_{s}}{\omega r} \frac{B_{p}}{B}\right)^{2} e^{i l q \theta} \frac{\partial^{2}}{\partial \theta^{2}} e^{-i l q \theta} \tilde{\phi}+\left(1-\frac{\omega_{*}}{\omega}+\frac{k_{y}^{2} c_{s}^{2}}{\Omega_{i}^{2}}-\frac{c_{s}^{2}}{\Omega_{i}^{2}} \frac{\partial^{2}}{\partial r^{2}}\right) \tilde{\phi} \\
& =F(\theta)\left\langle\frac{\omega-\omega_{* T}}{\omega-\omega_{D}+i \nu_{\text {eff }}}\right\rangle \bar{\phi}
\end{aligned}
$$

subject to appropriate boundary conditions, where ion temperature and resonant effects have been neglected, $\bar{\phi}$ is the bounceaveraged potential, and $F(\theta)$ is the local trapped particle fraction. In the absence of asymmetries, the solutions of the fluid equation are the standard harmonic components $e^{i m \theta}$. The introduction of small asymmetries should produce similarly small structural deviations, so we can expand the potential as $\tilde{\phi}(r, \theta)=\hat{\phi}(r, \theta) e^{i m \theta}$ where $\hat{\phi}$ is slowly varying poloidally. There are three sources of energetic asymmetry: (1) the bounceaverage, (2) the poloidally dependent trapped particle fraction 
$F(\theta)$, and $(3)^{\prime}$ the radial wavevector. In the limit of small phase shifts we neglect the first source and treat the differential equation. Since the fast poloidal structure is fixed by periodicity, the ion sound term is radially dependent and fixes the poloidally local radial structure at the standard shear eigenmodes. 3

The structure along the field line on the rational surface $(1 q=m)$ is determined by the equation

$0=\left(\frac{{ }_{s}{ }^{B} p}{\omega r B}\right)^{2} \frac{\partial^{2} \hat{\phi}}{\partial \theta^{2}}+\left(1-\frac{\omega *}{\omega}+\frac{k_{y}^{2} c_{s}^{2}}{\Omega_{i}^{2}}+\frac{i k_{y} c_{s}^{2}}{\omega \Omega_{i} L_{s}}(2 n+1)-F(\theta)\left\langle\frac{\omega-\omega_{\star T}}{\omega-\omega_{D}+i \nu_{\text {eff }}}\right\rangle \hat{\phi}\right.$

for the $n$-th radial eigenmode, where the local radial wavevector has been substituted as the fast variation and the boundary condition of outgoing energy has been assumed (i.e., reflections have been neglected). With the particular choice of $F(\theta)=F \cos ^{2}(\theta / 2)$. This equation reduces to the Mathieu form

$$
\frac{a^{2} \hat{\phi}}{d \theta^{2}}-(a+i b \cos \theta) \hat{\phi}=0
$$

where

$$
\begin{aligned}
& a \equiv-\left(\frac{\omega r B}{\dot{c}_{s}^{B} p}\right)^{2}\left(1-\frac{\omega_{\star}}{\omega}+\frac{k_{y}^{2} c_{s}^{2}}{\Omega_{i}^{2}}+\frac{i k_{y} c_{s}^{2}}{\omega_{i} L_{s}}(2 n+1)-\frac{i F}{2} I\right), \\
& b \equiv\left(\frac{\omega r B}{c_{s}{ }^{B} p}\right)^{2} \frac{F}{2} I,
\end{aligned}
$$


and only energetic asymmetries have been included (i.e., $b=$ real, $\left\langle\omega-\omega_{* T} / \omega-\omega_{D}+i \nu_{\text {eff }}\right\rangle \equiv i I, I=$ real).

For small values of energetic asymmetry $b$, a consistent even solution periodic in $2 \pi$ can be found with purely real eigenvalue

$$
a=-\frac{b^{2}}{2}+\cdots
$$

and eigenfunction

$$
\hat{\phi} \sim 1-i b \cdot \cos \theta+\ldots .
$$

Since the linear growth rate of the mode is determined by the shear, the poloidally averaged local energy influx from the trapped particles, and the imaginary part of the eigenvalue $a$, for these low b cases, the overall growth rate is given by the poloidally averaged local growth rate with the only effect of the asymmetry being: an increase of the real frequency? due to the ion sound term. Treatment of Eq. (3) shows that the $\cos \theta$ component of $|\hat{\phi}|^{2}$ is proportional to the imaginary part of a, so that for these cases of small $b$ there is no peaking in the region of larger local growth. However, for larger b the eigenvalue a has an imaginary part since the continued fraction solution breaks down due to a double root (the merging of modes $a=0,-1$ for $b=0.0$ ) at the value of $b=0.7343843$ and the two roots become complex conjugates for larger $b .5$ 
Since a becomes complex, localization occurs and there is an enhancement (diminution) of the growth rate for solutions peaked in the region of maximum (minimum) local growth rate. Table I gives the results of numerical computations for the most unstable root.

The expression for the mode structure for small asymmetries can be derived from basic principles. For the mode to be uniform, the energy must propogate along the parallel arc length in an asymmetric growth time,i.e., $\gamma_{1} \tau_{n}=1$,.. where, $\gamma_{1}$ is that component of the growth which is odd about $\theta=\pi / 2$ and $\tau_{n}=L_{n} / v_{g}$ where $L_{\|}$ is the parallel arc length and $v_{g "}$ the parallel group velocity. Substitution yields $k_{n n} L_{n} b$, in agreement with the small-b solution for the mode structure,. [see Eq. (7)]. The transition to the peaked profile occurs near that value of asymmetry such that $k_{11} L_{n} \sim 1$, which correctly suggests a coupling with another mode. Using the expression for $b$, a necessary condition for the onset of poloidal peaking due to the trapped electron growth term in the tokamak ordering may be obtained.' since for nominal values of temperature gradients and small ion gyroradii the magnitude of the energy average term I is limited to roughly 0.2 and the average asymetric component of the trapping fraction is roughly $(2 \mathrm{a} / \mathrm{k})^{\frac{1}{2}} 4 / 3 \pi$, for significant amplitude variation

$$
m>\frac{2.47}{q} \frac{\Omega_{i} a}{c_{s}}\left(\frac{a}{R}\right)^{3 / 4} .
$$


For the neglect of coupling and reflections at neighboring mode-rational surfaces, the radial wavelength must be less than the inter-surface distance, i.e.,

$$
m<\frac{\Omega_{i} a}{c_{s}}\left(\frac{r}{a} \frac{1}{q^{1} \cdot R}\right)^{\frac{1}{2}}
$$

Thus, for the compatibility between the existence of a poloidally peaked mode and the neglect of reflections and additional resonant damping

$$
q>q_{0} \equiv 6.11\left(\frac{0.2}{I}\right)\left(\frac{a}{R}\right)^{\frac{1}{2}}>6.11\left(\frac{a}{R}\right)^{\frac{1}{2}}
$$

In summary, for $q<q_{0}$ the integral equation nature of the problem can be ignored if modes are localized between mode-rational surfaces. Conversely, the introduction of coupling at other surfaces because of overlap réquires an integral equation treatment for $q>q_{0}$. The neglect of the parallel energy propagation in the variational principle seems justified only for large values of the poloidal mode number (large frequency) or $q$ (large parallel arc length).

\section{ACKNOWLEDGMENTS}

The author would like to thank Professor E. A. Frieman and Drs. M. Okabayashi, J. A. Schmidt, and W. M. Tang for insightful discussions during the course of this work and Mr. H. Fishman for the numerical computation of the eigenmodes.

This work was supported by United States Energy Research and Development Administration Contract E(11-1)-3073. 
REFERENCES

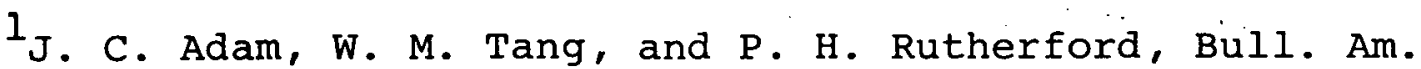
Phys. Soc. 20, 1288 (1975).

2W. Horton; Jr., D. W. Ross, W. M. Tang, H. L.:Berk;

E. A. Frieman, R. E. LaQuey, R. V. Lovelace, S. M. Mahajan,

M. N. Rosenbluth, and P. H. Rutherford, Plasma Physics and

Controlled Nuclear Fusion Research "(IAEA, Vienna 1975). Vol. 1, p. 541 .

3. L: D. Pearlstein and H. L. Berk, Phys. Rev. Lett. 23, 220 (1969).

${ }^{4}$ B. B. Kadamtsev and O. P. Pogutse, Dokl. Akad. Nauk SSSR 186, 553.(1969). [Sov. Phys. - Dokl. 14, 470 (1969)].

${ }^{5}$ H. P. Mulholland and S. Goldstein, Phil. May. 8,834 (1929). 


\begin{tabular}{lcc}
$\frac{b}{1.00}$ & $\frac{a(\text { Real) }}{a(\text { Imaginary) }}$ \\
0.01 & 0.0 & 0.0 \\
0.02 & -0.00005 & 0.0 \\
0.04 & -0.00020 & 0.0 \\
0.08 & -0.00080 & 0.0 \\
0.16 & -0.00321 & 0.0 \\
0.20 & -0.01295 & 0.0 \\
0.30 & -0.02036 & 0.0 \\
0.40 & -0.04694 & 0.0 \\
0.50 & -0.08662 & 0.0 \\
0.60 & -0.14319 & 0.0 \\
0.70 & -0.22548 & 0.0 \\
0.80 & -0.36820 & 0.0 \\
1.60 & -0.52625 & -0.21746 \\
2.40 & -0.59984 & -0.99279 \\
3.20 & -0.70530 & -1.64067 \\
4.00 & -0.82121 & -2.30637 \\
\hline 00 & -0.93132 & -2.99545
\end{tabular}

